\title{
Control Techniques for Multiple Current Source GTO Converters
}

\author{
M. C. Chandorkar, Student Member, IEEE, D. M. Divan, Senior Member, IEEE, \\ and R. H. Lasseter, Fellow, IEEE
}

\begin{abstract}
This paper presents the control techniques for a multiple current source GTO converter in a high-power environment. The output current space vector diagram for the multiple converter is presented. The important issue of dc current sharing between the two bridges is discussed, and a novel current sharing control scheme based on the use of redundant switch combinations is presented. Harmonic issues are discussed. The ranges of independent control of real and reactive power for different dc power sources are presented.
\end{abstract}

\section{INTRODUCTION}

$\mathbf{I}$ $\mathrm{N}$ RECENT years, high power inverter topologies have attracted considerable attention as interfaces between dc energy storage systems and the ac power transmission and distribution systems. In this regard, current source inverters (CSI) using gate turn-off (GTO) thyristors are of special interest, since they permit four quadrant operation. During forced commutation, a GTO CSI needs to have a mechanism to absorb the energy trapped in the line inductance. This is usually done by connecting capacitors between the ac lines [1]. The mechanism of occurrence of the over-voltage and a method of its suppression is also discussed in [1].

From the foregoing discussion, it is seen that a primary task of the GTO CSI is to achieve control of both the magnitude of the output current vector, and its position relative to the ac system voltage. The control of these two quantities preferably should be independent over as wide a range of inverter operation as possible. On the dc side, it is equally important to ensure that the inverter presents the proper input characteristics to the dc source, possibly by the use of closed loop control.

In high-power inverter topologies, it is not advisable to employ pulse width modulation (PWM), at least around the inverter nominal operating point, due to switching loss concerns. However, at high-power levels, harmonic issues gain in importance. To reconcile these conflicting demands, various solutions have been proposed in the past. For example, [2] described an early use of parallel connection of six-step inverters by means of phase-shifting transformers. The resulting multi-stepped inverter output voltage waveform had a reduced harmonic content compared to the output of a single six-step

Paper IPCSD 94-56, approved by the Industrial Power Converter Committee of the IEEE Industry Applications Society for presentation at the Power Conversion Conference-Yokohama 1993, Yokohama, Japan, April 19-21. This work was supported by the Wisconsin Electric Machines and Power Electronics Consortium (WEMPEC). Manuscript released for publication July $1,1994$.

The authors are with the Department of Electrical and Computer Engineering, University of Wisconsin, Madison, WI 53706 USA

IEEE Log Number 9406622.

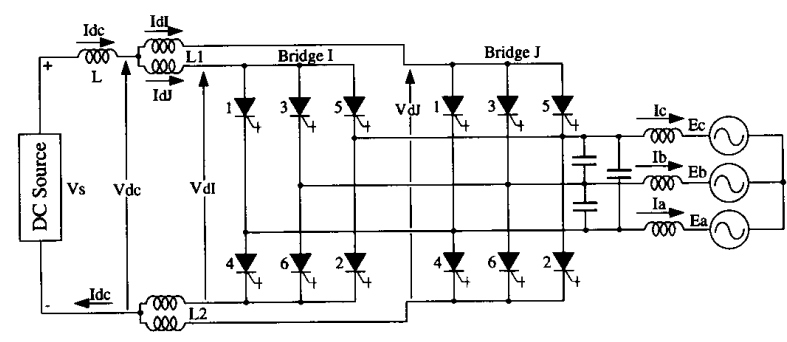

Fig. 1. Multiple CSI topology.

inverter. Inverter paralleling also increased the overall power rating of the combined system.

More recently, the so-called multi-level inverter topologies have been proposed. In the voltage source inverter (VSI) domain, [3] proposed the neutral-point clamped inverter (NPC VSI). In the current source inverter (CSI) domain, a multilevel inverter topology was proposed at an even earlier date by [4], and was called a multiple current source inverter.

Subsequently, [5] proposed the use of the multiple CSI of [4] for induction machine control. This consisted of two CSI modules, and was thus called a Dual Current Source Inverter. Further, [6] proposed its use for interfacing a superconducting magnetic energy storage (SMES) coil to a power system, and [7] extended the concept to the use of multiple modules in place of two.

The essential topology of the multiple GTO CSI is given in Fig. 1. The important topological and operational features of the converter have been discussed in [4] and [5], and are only briefly mentioned here. The intercircuit reactors $L_{1}$ and $L_{2}$ permit the division of the total dc current $I_{d c}$ into the two GTO bridge circuits, and so the maximum current in a device at any time is $I_{d c} / 2$. It is possible to control the fundamental magnitude of the ac current by correctly controlling the relative phase shift between the two bridges, without PWM. It is also possible to control its phase relative to the ac voltage.

The central aim of this paper is to bring into focus the control aspects of the multiple CSI, and to discuss in detail its advantages and limitations when used in a power system environment.

\section{Control Requirements}

On the dc side, it must be ensured that equal average $\mathrm{dc}$ currents exist in the two bridges (see Fig. 1). The intercircuit reactors $L_{1}$ and $L_{2}$, along with a proper GTO firing algorithm, permit this equal current division. To avoid dc current drift, 
the firing algorithm must ensure that the steady state average voltage across each intercircuit reactor is just enough to compensate for resistive drops in that reactor. It must also ensure that the proper dc voltage, $V_{d c}$ in Fig. 1, appears on the $\mathrm{dc}$ bus.

On the ac side, the firing algorithm must ensure that the ac current has the desired fundamental amplitude, and has the desired phase relative to the ac voltage.

The extent to which these four basic control requirements can be met depends on the nature of the dc source (constant current or constant voltage). A constant dc current source has no essential restrictions on the dc voltage across it, and $V_{d c}$ can vary over a wide range, both positive and negative. Thus, the ac real power $P$ is determined by the value of $V_{d c}$, and can be either positive or negative. For example, this feature could be used to discharge or charge a SMES coil.

A constant dc voltage source imposes a constraint on the value of $V_{d c}$, and ac power control can be achieved by controlling the value of the dc current $I_{d c}$. It is well known that power flow in this case is unidirectional. The nature of the dc source affects not only the control of ac real power, but also the range over which independent control of ac real power $P$ and reactive power $Q$ can be achieved.

The vector representation detailed in the next section provides a very convenient means to implement the control schemes. Later, a systematic switching algorithm which exploits the redundancies in the switch combinations to achieve equal dc current sharing between the bridges is described.

\section{VECTOR REPRESENTATION OF OUTPUT CURRENTS}

It will prove to be convenient to transform the ac line currents, vectorially, to the $d-q-n$ reference frame, after the manner of Park [8]. In this framework, the currents are transformed according to the relations:

$$
\begin{aligned}
i_{d} & =\frac{2}{3}\left[i_{a} \sin (\theta)+i_{b} \sin \left(\theta-\frac{2 \pi}{3}\right)+i_{c} \sin \left(\theta+\frac{2 \pi}{3}\right)\right] \\
i_{q} & =\frac{2}{3}\left[i_{a} \cos (\theta)+i_{b} \cos \left(\theta-\frac{2 \pi}{3}\right)+i_{c} \cos \left(\theta+\frac{2 \pi}{3}\right)\right] \\
i_{n} & =\frac{2}{3}\left(\frac{1}{\sqrt{2}} i_{a}+\frac{1}{\sqrt{2}} i_{b}+\frac{1}{\sqrt{2}} i_{c}\right) .
\end{aligned}
$$

For the transformation required here, the angle $\theta$ can be chosen to be an arbitrary value convenient for the presentation. If $\theta$ is chosen to be 0 , then the transformation becomes:

$$
\begin{aligned}
i_{d} & =\frac{2}{3}\left(-\frac{\sqrt{3}}{2} i_{b}+\frac{\sqrt{3}}{2} i_{c}\right) \\
i_{q} & =\frac{2}{3}\left(i_{a}-\frac{1}{2} i_{b}-\frac{1}{2} i_{c}\right) \\
i_{n} & =\frac{2}{3}\left(\frac{1}{\sqrt{2}} i_{a}+\frac{1}{\sqrt{2}} i_{b}+\frac{1}{\sqrt{2}} i_{c}\right) .
\end{aligned}
$$

In these equations, $i_{a}, i_{b}, i_{c}$ are the instantaneous values of the three line currents. If the sum of the currents is zero, as it is assumed here, $i_{n}$ is zero.

The vector representation provides a convenient means for considering the combined action of the two bridges. It enables the analytical description of the output currents, and facilitates the development of control schemes for the

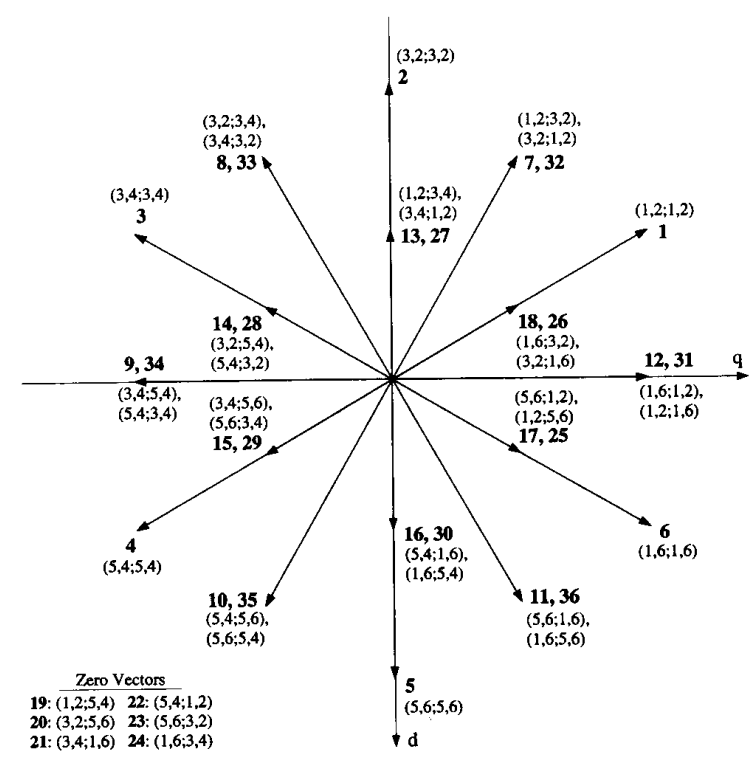

Fig. 2. Multiple CSI vectors.

inverter. Fig. 2 shows all the 19 possible output current vectors and the 36 switch combinations that generate these vectors. The redundancy between the switch combinations is readily apparent from Fig. 2.

In Fig. 2, the numbers in bold denote the switch combination number. The numbers in parentheses denote the conducting switches which result in the vector. For example, switch combination number $\mathbf{8}$ is achieved by the switches $(3,2 ; 3,4)$, in which switches 3 and 2 from Bridge $I$ and switches 3 and 4 from Bridge $J$ conduct.

The method of selection of the vectors determines the output current waveform. Fig. 2 shows that apart from the zero vector, there are three distinct classes of vectors. Class I consists of the "large" vectors $\mathbf{1}, \mathbf{2}, \ldots$, Class II consists of the "medium" vectors $7,8, \ldots$, and Class III consists of the "small" vectors $13,14, \ldots$, as in Fig. 2. When only the vectors of Class $I$ are used in sequence, the output ac current waveform is the standard six-step waveform with a maximum value of $I_{d c}$. Multi-stepped waveforms are obtained by the use of the other vector classes. It is possible to control the magnitude of the fundamental component of the output current by changing the time for which each vector class is chosen.

An added flexibility is provided by the fact that each vector in Classes II and III can be synthesized by two distinct switch combinations. This means that the same output current waveform can be produced with two different voltages across the intercircuit reactors. This fact can be used very effectively to enforce equal dc currents in the two bridges, as will be described in Section VI below.

\section{AC POWER FLOW}

This section considers the flow of real power $P$ and reactive power $Q$ in the ac system. It is emphasized here that for power system applications, the real and reactive power flows in the ac power sources are important from the points of view of 


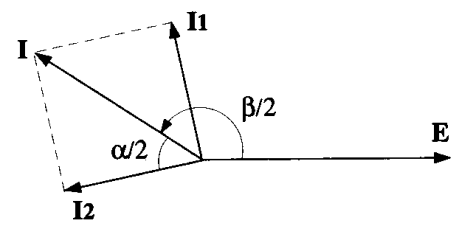

Fig. 3. AC voltage and CSI current.

load flow calculations, stability analysis and control. For these reasons, it important to consider the $P$ and $Q$ flows in the multiple converter topology.

The expressions for $P$ and $Q$ can be obtained on the basis of the vectors depicted in Fig. 3, which shows various vectors in the stationary $d-q$ reference frame at some instant of time. $\mathbf{E}$ is the ac system voltage vector. $\mathbf{I}_{1}$ and $\mathbf{I}_{2}$ are the current vectors of the two bridges. $I$ is the vector current provided to the ac system. The definition of the angles $\alpha$ and $\beta$ in Fig. 3 provides a means to develop very elegant control schemes. The angle $\alpha$ determines the magnitude of the ac current. The power factor angle $\phi$ is equal to $\beta / 2$. With these angle definitions, the expressions for $P, Q$ and $V_{d c}$ are:

$$
\begin{aligned}
P & =\frac{3}{2 \pi} E I_{d c}\left[\cos \left(\frac{\beta+\alpha}{2}\right)+\cos \left(\frac{\beta-\alpha}{2}\right)\right] \\
Q & =\frac{3}{2 \pi} E I_{d c}\left[\sin \left(\frac{\beta+\alpha}{2}\right)+\sin \left(\frac{\beta-\alpha}{2}\right)\right] \\
V_{d c} & =\frac{3}{2 \pi} E\left[\cos \left(\frac{\beta+\alpha}{2}\right)+\cos \left(\frac{\beta-\alpha}{2}\right)\right] .
\end{aligned}
$$

In these equations, $E$ is the magnitude of the ac system line-line voltage, and $I_{d c}$ is the dc current shown in Fig. 1. It is convenient to define the following two quantities:

$$
\begin{aligned}
& a=\cos \left(\frac{\beta+\alpha}{2}\right)+\cos \left(\frac{\beta-\alpha}{2}\right) \\
& b=\sin \left(\frac{\beta+\alpha}{2}\right)+\sin \left(\frac{\beta-\alpha}{2}\right)
\end{aligned}
$$

The values of $\alpha$ and $\beta$ can be obtained by solving (4) and (5). These are found to be:

$$
\begin{aligned}
& \alpha=\cos ^{-1}\left(\frac{a^{2}+b^{2}-2}{2}\right) \\
& \beta=\cos ^{-1}\left(\frac{a^{2}-b^{2}}{a^{2}+b^{2}}\right)+2 \pi .
\end{aligned}
$$

Equations (1), (2), and (3) indicate the range over which $P$ and $Q$ can be controlled independently for fixed ac voltage $E$. This range is critically determined by the nature of the $\mathrm{dc}$ source.

With a $d c$ current source, the dc voltage across the source can vary over a wide range. In principle, the quantities $a$ and $b$ in (4) and (5) can vary between +2 and -2 . Thus, $P$ and $Q$ can simultaneously take any value within a circular boundary in the $P-Q$ plane. This is shown in Fig. 4(a). The boundary expands with increasing $I_{d c}$. It is to be noted that in Fig. 4, $P$ and $Q$ delivered by the inverter to the ac power system is considered negative.

With a $d c$ voltage source, the situation is more complicated. The presence of the voltage source requires that to ensure voltseconds balance in the inductor $L$, the steady-state average

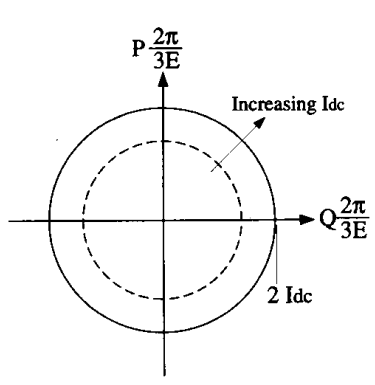

(a)

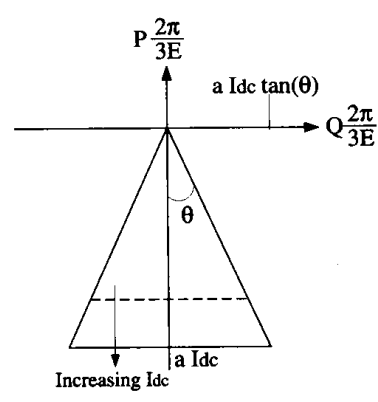

(b)
Fig. 4. Regions of $P$ and $Q$ control. (a) With dc current source. (b) With dc voltage source.

voltage on the inverter dc bus, $V_{d c}$, must equal the dc source voltage, $V_{s}$, if the resistive voltage drop across $L$ is neglected. This imposes a rather severe restriction on the range of independent $P$ and $Q$ operation. For a fixed ac voltage, (3) and (4) indicate that $a$ must be a constant, dictated by the value of the dc source voltage $V_{s}$. The range of control is thus characterized by the range over which $b$ can be varied, keeping $a$ constant. The region of operation with a dc voltage source is shown in Fig. 4(b). For a given dc current $I_{d c}$, the steady-state ac power $P$ is constant. The reactive power $Q$ can be altered along the horizontal line shown in Fig. 4(b). Thus, the region of operation is simply a line parallel to the $Q$-axis.

\section{AC CURRENT CONTROL}

The two control angles $\alpha$ and $\beta$ were defined in the previous section. It is apparent from Fig. 3 that the magnitude of the ac current is controlled by the angle $\alpha$. The fundamental ac current magnitude is controlled from maximum to zero by controlling $\alpha$ in the range $0 \leq \alpha \leq \pi$. The maximum ac current is obtained when $\alpha=0$. Over this range, the ac current waveform changes, and so does the harmonic spectrum of the ac current.

Fig. 5 shows the construction of the line current $i_{a}$ for $0 \leq \alpha \leq \pi / 3$. The spectrum of this waveform can be easily obtained by considering odd symmetry, and setting up a Fourier sine series. The line current can then be represented as:

$$
\begin{aligned}
i_{a}(t) & =\sum_{n=1}^{\infty} b_{n} \sin (n \omega t) \\
b_{n} & =\frac{4 I_{d c}}{n \pi} \cos \left(\frac{n \pi}{6}\right) \cos \left(\frac{n \alpha}{2}\right) \\
n & =1,3,5, \ldots
\end{aligned}
$$

In these equations, $I_{d c}$ is the dc current as shown in Fig. 1 , and $\omega$ is the fundamental angular frequency $\left(377 \mathrm{rs}^{-1}\right.$ for $60 \mathrm{~Hz}$ ). Although (8) and (9) are derived for the case $0 \leq \alpha \leq \pi / 3$ (see Fig. 5), it can be shown that the same equations hold for the entire range of $\alpha$.

Fig. 6 gives the variation of the fundamental amplitude of the ac line current, and the amplitudes of the 5th, 7th, 11th, 13th, and 17th harmonic components, as functions of $\alpha$. In this figure, equal sharing of the dc current by the bridges is assumed. The figure indicates the variation possible in 


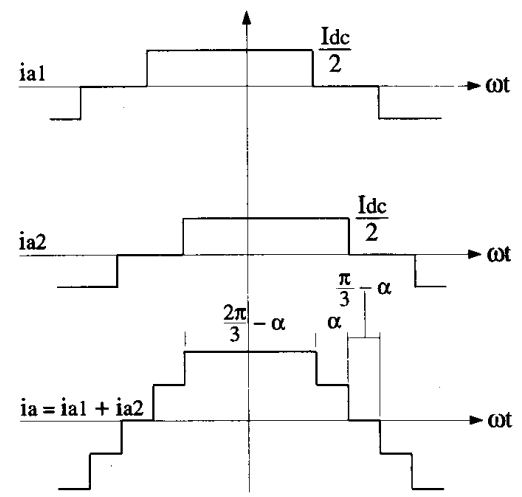

Fig. 5. AC line current.

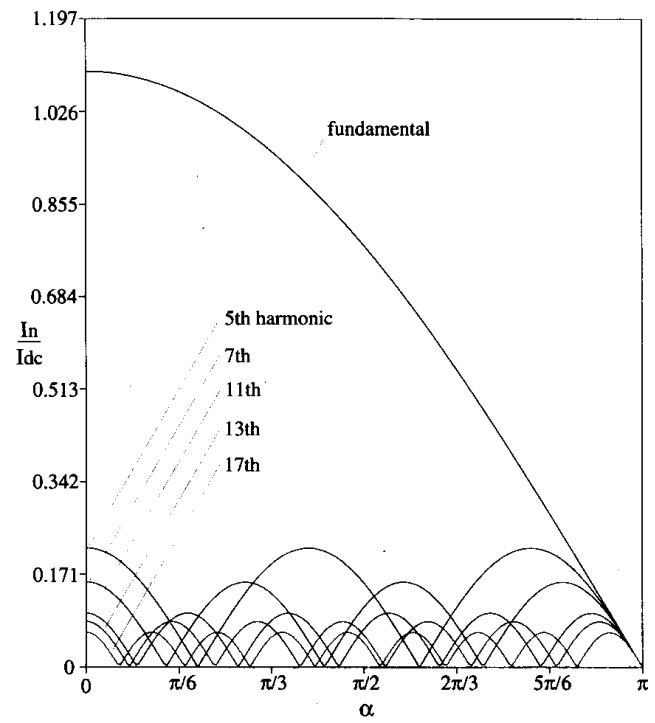

Fig. 6. AC line current harmonics.

the fundamental amplitude. Operation at large values of $\alpha$ results in larger harmonic content relative to the fundamental. Thus, in general, it is advisable to choose a nominal operating point with $\alpha$ around $\pi / 5$, where the 5th harmonic has zero amplitude, and other harmonics have low amplitudes.

As described above, the angle $\alpha$ is used to control the magnitude of the fundamental frequency ac current. The position of the ac current relative to the ac voltage is likewise controlled by the angle $\beta$, shown in Fig. 3. The angle $\beta$ is a measure of the delay in generating the switch firing pulses relative to the ac voltage. In fact, the angle $\beta / 2=\phi$ is the displacement power factor angle. The range of values possible for $\beta$ is $\pi \leq \beta \leq 3 \pi$. In the range $\pi \leq \beta \leq 2 \pi$, the inverter takes reactive power $Q$ from the ac power system. In the range $2 \pi \leq \beta \leq 3 \pi$, the inverter gives reactive power to the ac power system.

The link between the control angle $\alpha$ and the vector diagram of Fig. 2 will now be established. The output current vectors shown in Fig. 2 are chosen sequentially in the anti-clockwise direction. There are 19 current vectors, but not all are chosen. The value of $\alpha$ determines which vectors are chosen.

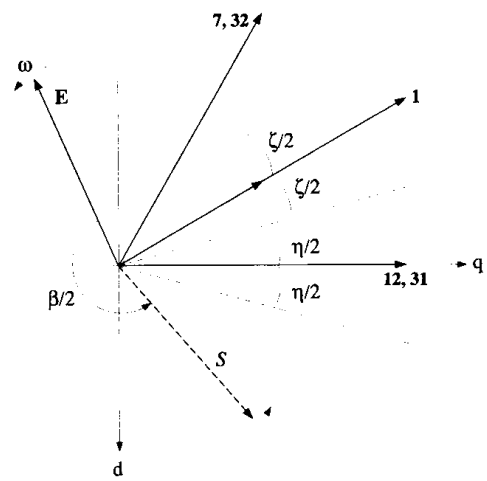

Fig. 7. Current vector control.

TABLE I

\begin{tabular}{|l|c|c|}
\hline Angles $\rightarrow$ & $\eta$ & $\zeta$ \\
\hline $0 \leq \alpha<\pi / 3$ & $\alpha$ & $\pi / 3-\alpha$ \\
\hline$\pi / 3 \leq \alpha<2 \pi / 3$ & $2 \pi / 3-\alpha$ & $\alpha-\pi / 3$ \\
\hline $2 \pi / 3 \leq \alpha<\pi$ & $\alpha-2 \pi / 3$ & $\pi-\alpha$ \\
\hline
\end{tabular}

For $0 \leq \alpha<\pi / 3$, the "large" vectors of Class I and the "medium" vectors of Class II (refer to Section III) are chosen in anti-clockwise sequence. Each Class I vector is chosen for a time corresponding to $\pi / 3-\alpha$, and each Class II vector is chosen for $\alpha$. Thus, for example, for $\alpha=0$, only the Class I vectors are chosen, and the inverter operation is the conventional six-step, resulting in maximum output current. For higher values of $\alpha$, Class II vectors are chosen for longer, and Class I vectors for shorter, durations. The ac fundamental current magnitude decreases.

For $\pi / 3 \leq \alpha<2 \pi / 3$, the "medium" vectors of Class II and the "small" vectors of Class III are chosen in anti-clockwise sequence. Each Class II vector is chosen for $2 \pi / 3-\alpha$, and each Class III vector is chosen for $\alpha-\pi / 3$.

For $2 \pi / 3 \leq \alpha \leq \pi$, the "small" vectors of Class III and a zero vector are chosen. Each Class III vector is chosen in anticlockwise sequence for $\pi-\alpha$, and the zero vector is chosen for $\alpha-2 \pi / 3$.

Fig. 7 shows the scheme to achieve complete control of the ac current vector. For convenience, only a few of the 19 possible inverter current vectors are shown. The ac power system voltage vector is obtained by transforming the instantaneous values of the three phase ac voltages to the stationary $d-q$ reference frame. The resulting vector, $\mathbf{E}$, rotates as shown with an angular frequency $\omega$. The position reference for the desired inverter current vector is obtained by adding $\beta / 2$ to the instantaneous position of the ac voltage vector $\mathbf{E}$. The position of the desired inverter current vector is denoted by the line $S$.

To control the magnitude of the desired inverter current, the angles $\eta$ and $\zeta$ are defined (see Fig. 7), by Table I.

These angles define sectors around each inverter switching vector, as in Fig. 7. When the line $S$ enters a sector, the switching vector contained in the sector is chosen for output. 
There are multiple choices of switch combinations in each sector (see Fig. 2). These choices are made so as to achieve zero average voltage across the intercircuit reactors $L_{1}$ and $L_{2}$, thus ensuring equal dc current sharing between the two bridges. This is possible over the entire ranges of $\alpha$ and $\beta$, and the process is detailed in the next section.

\section{DC CURRENT BALANCE}

The correct choice of vectors can be made systematically as the line $S$ sweeps around anti-clockwise, so as to achieve dc current balance between the two bridges. This is done by choosing the vectors to ensure zero average voltage across each intercircuit inductor. The vectors chosen depend on the value of $\alpha$. The sequences of vectors chosen are given [see bottom of this page].

The essential idea behind dc current balance control is that for vector classes II and III, the same ac current vector can be generated by two distinct switch combinations. Thus, the current balance control on the dc side can be decoupled from the ac side. For example, for the vector $(\mathbf{7}, \mathbf{3 2})$ in Fig. 2 , there are two distinct switch combinations: $(1,2 ; 3,2)$ and $(3,2 ; 1$, $2)$. The combination $(1,2 ; 3,2)$ results in a voltage $E_{a b}$ across intercircuit reactor $L_{1}$. The combination $(3,2 ; 1,2)$ results in a voltage $E_{b a}$ across across $L_{1}$. These two combinations affect the dc currents $I_{d c I}$ and $I_{d c J}$ in opposite ways, but generate the same output ac current vector.

The above sequences indicate that the switching repeats after two cycles. The voltage across each intercircuit reactor then has a zero average value over $\frac{2}{3}$ of the ac voltage cycle of the power system. With these choices of the switching sequence, it is possible to operate over the entire ranges of the control angles $\alpha$ and $\beta$, and still achieve dc current balance. Tight closed-loop control of the difference between $I_{d c I}$ and $I_{d c J}$ ensures that the two bridge currents do not deviate from each other. This is achieved by making the correct choice of the switch combination every time a new output current vector is chosen.

It must be noted here that the above sequences are not unique. Other switching sequences that ensure dc current balancing are possible, and an appropriate sequence might be chosen that satisfies some specified criterion.

It can be shown that the sequences given above result in an average dc voltage, $V_{d c}$ in Fig. 1, as given by (3), provided that the intercircuit reactors have equal value. In that case, the lowest voltage harmonic present in $V_{d c}$ is the 6th. The amplitude of the harmonic content of $V_{d c}$ depends on the control angles $\alpha$ and $\beta$.

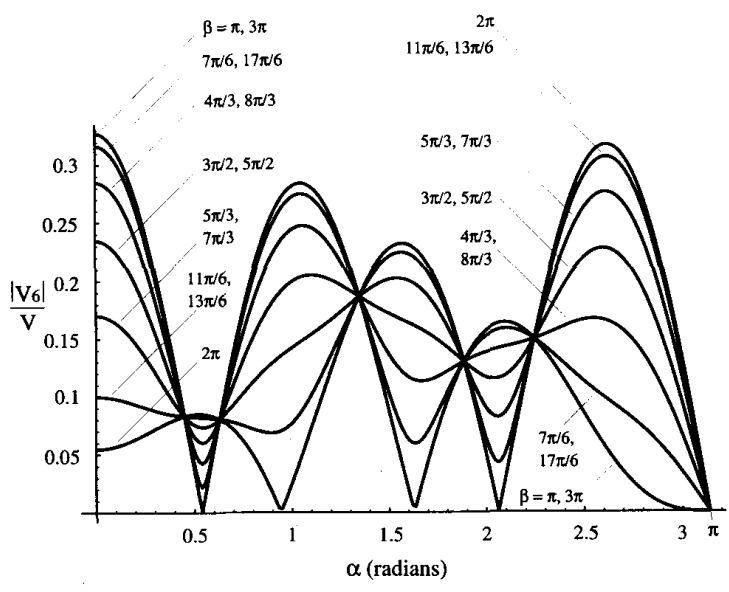

Fig. 8. Sixth harmonic in $V_{d c}$ versus $\alpha$.

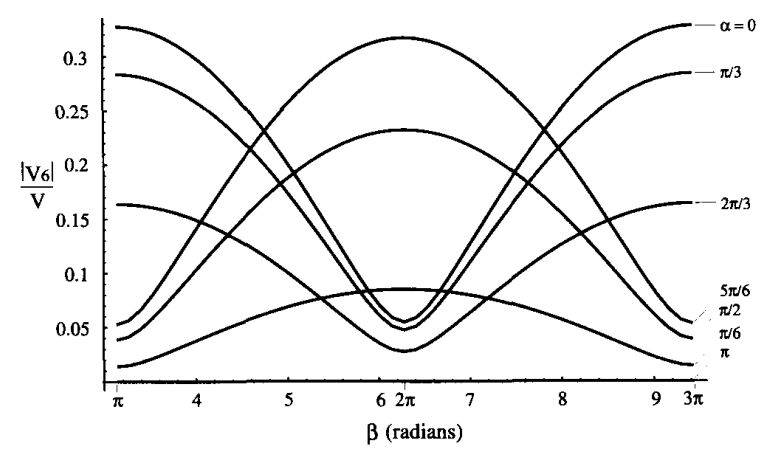

Fig. 9. Sixth harmonic in $V_{d c}$ versus $\beta$.

Fig. 8 shows the normalized amplitude of the 6th harmonic component of the dc voltage $V_{d c}$, as a function of $\alpha$. The normalizing factor is $V$, the peak value of the ac system line-line voltage. The curves in Fig. 8 are plotted with the control angle $\beta$ as a parameter. It is seen that in the range of $\alpha$ between 0.45 and $0.65 \mathrm{rad}$, the 6th harmonic has low values for any given $\beta$. It would thus be desirable to set the nominal $\alpha$ operating point of the inverter to be within this range.

Fig. 9 shows the normalized amplitude of the 6th harmonic component of $V_{d c}$ as a function of $\beta$, with $\alpha$ as a parameter. The normalizing factor is the same as described above. The curves are symmetric about $\beta=2 \pi$, and have either maxima or minima, depending on the value of $\alpha$. However, if the nominal value of $\alpha$ is taken to be $\pi / 6$, the normalized amplitude of the $6 t h$ harmonic is less than $\approx 0.085$, regardless of the value of $\beta$.

$$
\begin{aligned}
& 0 \leq \alpha<\pi / 3 \\
& 1,7,2,8,3,34,4,35,5,11,6,12,1,32,2,33,3,9,4,10,5,36,6,31,1, \ldots \\
& \pi / 3 \leq \alpha<2 \pi / 3 \\
& 26,7,13,8,14,34,29,35,30,11,17,12,18,32,27,33,28,9,15,20,16,36,25,31,26 \ldots \\
& 2 \pi / 3 \leq \alpha<\pi \text { : }
\end{aligned}
$$$$
26,22,13,23,14,21,29,22,30,20,17,21,18,19,27,20,28,24,15,19,16,23,25,24,26, \ldots
$$ 


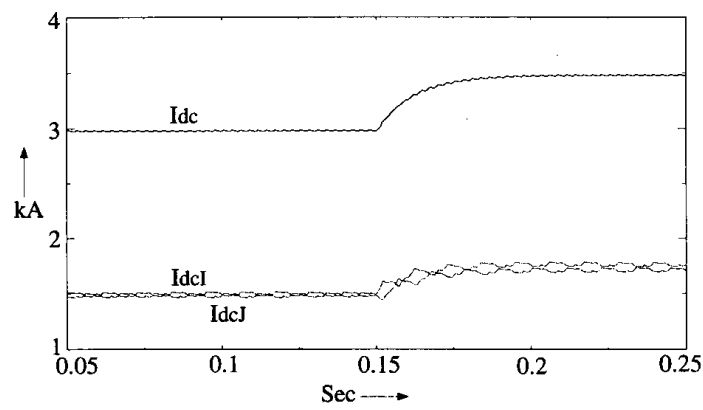

Fig. 10. DC current change and current balance.

The effectiveness of the current balance method detailed above is demonstrated by a simulation example in the next section.

\section{EMTP SIMULATIONS}

The multiple CSI, along with the vector control scheme and current balance method detailed above, has been modeled using the Electromagnetic Transients Program (EMTP). The dc source has been considered to be separately a voltage source and a current source. Some waveforms obtained from the simulations are presented below.

With a $d c$ voltage source, the steady state average value of $V_{d c}$ (see Fig. 1) is constrained to be equal to the dc source voltage. A very convenient control scheme to meet this constraint is to have closed loop control of the dc current. Thus, there is no need to have explicit knowledge of the dc source voltage value $V_{s}$. The closed loop controller operates on the error between the set value of $I_{d c}$, and the actual value, obtained by measurement. This also naturally controls the ac real power $P$. The dc current controller determines the value of $a$ (refer to (4)). On the ac side, the closed loop control is on the reactive power $Q$. The controller acts on the error between the set and actual values of $Q$, and determines the value of $b$ (refer to (5)). Equations (6) and (7) are then used to compute the values of the control angles $\alpha$ and $\beta$. These values of $\alpha$ and $\beta$ are used to sequentially select the appropriate current vectors, and generate the correct switch firing pulses as described in the previous sections.

Fig. 10 shows the response of the dc currents to a change in the dc current set-point from $3 \mathrm{kA}$ to $3.5 \mathrm{kA}$. The dc current controller accomplishes this task. The choice of proper current switching vectors to achieve current balancing ensures that $I_{d c}$ is shared equally by the two bridges, such that $I_{d c I}=I_{d c J}$, on average, even transiently. This demonstrates the effectiveness of the current balancing scheme detailed above.

With a dc current source, the dc voltage can vary over a wide range. The control is now on the ac side quantities, $P$ and $Q$. The error in real power $P$ is used to determine the value of $a$ (refer to (4)). Likewise, the error in reactive power $Q$ is used to determine the value of $b$ (refer to (5)). From $a$ and $b$, the values of the control angles $\alpha$ and $\beta$ are computed using (6) and (7). These are then used to select the appropriate vectors and generate the firing pulses as detailed in the previous two sections.

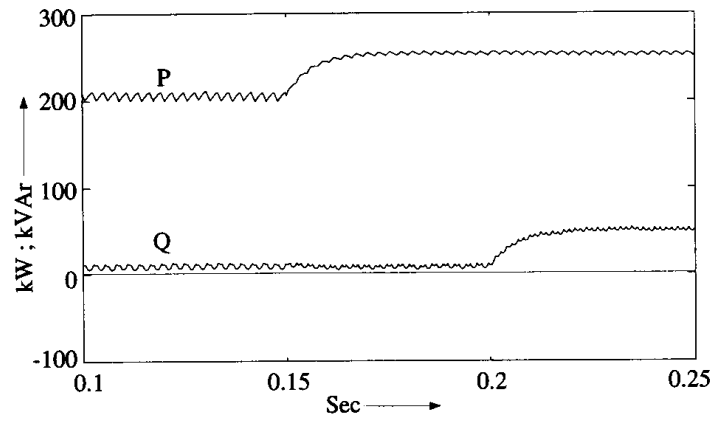

Fig. 11. $P$ and $Q$.

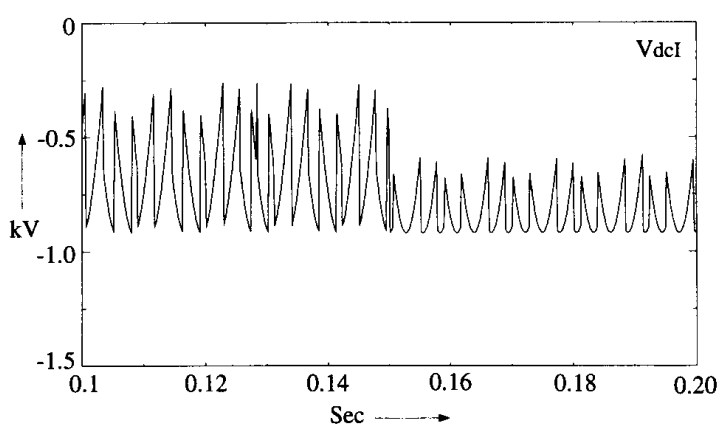

Fig. 12. DC voltage $V_{d c I}$.

Fig. 11 shows the change in $P$ and $Q$ in response to change in the set-points. At $0.15 \mathrm{~s}$, the $P$ set-point is changed from $200 \mathrm{~kW}$ to $250 \mathrm{~kW}$, and at $0.2 \mathrm{~s}$, the $Q$ set-point is changed from $10 \mathrm{kVAr}$ to $50 \mathrm{kVAr}$. The waveforms show excellent decoupling between the controls of these two quantities. The traces of Fig. 11 have been averaged to reduce ripple present in $P$ and $Q$, in order to better demonstrate the decoupling of the control of the two quantities.

Fig. 12 shows the dc voltage across Bridge I (see Fig. 1), when the $P$ set-point is changed from $200 \mathrm{~kW}$ to $250 \mathrm{~kW}$ at $0.15 \mathrm{~s}$. The figure shows an increase in the dc bus voltage in response to the increase in power command. It is to be noted that the waveform is such as to ensure zero average voltage across the intercircuit reactors $L_{1}$ and $L_{2}$.

Fig. 13 shows the total dc voltage $V_{d c}$ (see Fig. 1), for the same change in the $P$ set-point as above. The increase in the average value of $V_{d c}$ after $0.15 \mathrm{~s}$ is readily apparent. Also, it is noted that the harmonic of the lowest order in this waveform is the 6th harmonic.

Fig. 14 shows the ac line current $i_{a}$, for the same change in the $P$ set-point as above. The change in the wave-shape at $0.15 \mathrm{~s}$ is apparent. Clearly, the amplitude of the fundamental ac current increases after $0.15 \mathrm{~s}$.

\section{CONCLUSION}

This paper has brought into focus the major issues related to the control of the Multiple CSI in a power system environment. For this purpose, the vector diagram of the multiple CSI has been presented. The issue of current balancing between the two bridges of the converter has been resolved by making use 


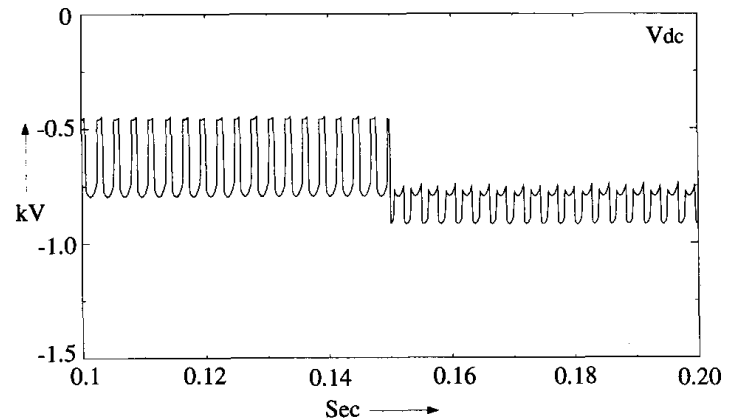

Fig. 13. DC voltage $V_{d c}$.

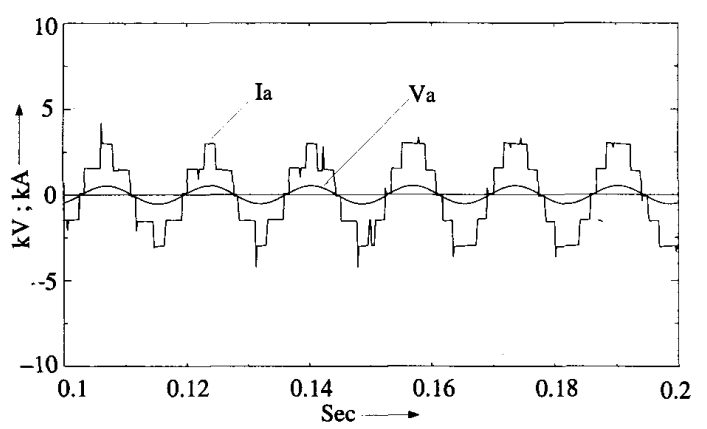

Fig. 14. AC line current and voltage.

of the redundant switch combinations which result in the same output current vector. This has been achieved without the need to use pulse width modulation (PWM) of the output current.

The regions of independent control of $P$ and $Q$ have been presented for both a current and a voltage dc source. It has been demonstrated that a dc voltage source severely restricts the range of operation of the multiple CSI. This would probably make the topology unsuitable for operation with many voltagetype energy sources. However, the topology could be useful in applications like grid-connected photovoltaic systems. The multiple CSI has very good performance with a current source, making it attractive for application with, for example, SMES. The dependence of ac and dc side harmonics on the operating point has been presented.

The multiple CSI circuit and the associated vector control schemes have been simulated using the Electromagnetic Transient Program (EMTP). The results of the simulations have been presented to validate the control mechanisms.

\section{ACKNOWLEDGMENT}

Discussions with Sasan Jalali regarding dc current balancing are gratefully acknowledged.

\section{REFERENCES}

[1] M. Hombu et al., "A new current source GTO inverter with sinusoidal output voltage and current," IEEE Trans. Ind. Applicat., Sept./Oct. 1985, pp. 1192-1198.

[2] C. W. Flairty, "A $50 \mathrm{kVA}$ adjustable-frequency 24-phase controlledrectifier inverter," AIEE Ind. Electron. Symp., Boston, MA, Sept. 20-21, 1961.

[3] A. Nabae, I. Takahashi, and H. Akagi, "A new neutral point clamped PWM inverter," IEEE Trans. Ind. Applicat., Sept./Oct. 1981, pp. 518-523.

[4] A. Nabae, T. Shimamura, R. Kurosawa, "A New Multiple Current Source Inverter," Conf. Rec. IEEE Int. Power Semiconductor Converter Conf., 1977, pp. 200-204.

[5] M. Hombu, S. Ueda, K. Honda, and A. Ueda, "A multiple current source GTO inverter with sinusoidal output voltage and current," Conf. Rec., IEEE Ind. Applicat. Soc. Ann. Mtg., 1987, pp. 600-606.

[6] R. H. Lasseter and S. G. Jalali, "Power conditioning systems for superconductive magnetic energy storage," IEEE Trans. Energy Conversion, vol. 6 , no. 3, 1991, pp. 381-397.

[7] Z. Zhang and B. T. Ooi, "Multi-modular current source SPWM converters for superconducting magnetic storage system," IEEE PESC Conf. Rec., 1992, pp. 561-568.

[8] R. H. Park, "Two-reaction theory of synchronous machines - generalized method of analysis - Part 1," Trans. AIEE, vol. 48, pp. 716-727, 1929.

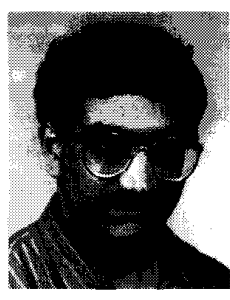

Mukul C. Chandorkar (M'85-S'90) received the B.Tech, degree in electrical engineering from the Indian Institute of Technology, Bombay, in 1984, and the M.Tech. degree in electrical engineering from the Indian Institute of Technology, Madras, in 1987.

From 1984-1986, he was with Larsen and Toubro Limited, Bombay, working on the engineering of cement and chemical plants. He worked as a design engineer in the power electronics industry in India during 1988-1989. Since 1989, he has been working on the Ph.D. program in electrical and computer engineering at the University of Wisconsin, Madison. His primary technical interests are in power electronics applications to electric machines and power systems.

Deepakraj M. Divan (S'78-M'78-S'82-M'83-SM'91), for a photograph and biography, please see page 34 of this issue.

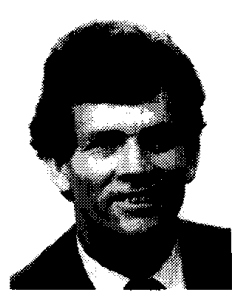

Robert H. Lasseter (M'74-SM'89-F'92) received the Ph.D. degree in physics at the University of Pennsylvania, Philadelphia, in 1971.

He was a Consultant Engineer at General Electric Company. He joined the University of Wisconsin, Madison, in 1980. His main interest is the application of power electronics to utility systems. 